\title{
Immuno-oncology in triple-negative breast cancer
}

\author{
Anne-Sophie Heimes, Marcus Schmidt \\ Department of Obstetrics and Gynecology, University Medical Center Mainz, Mainz 55131, Germany. \\ Correspondence to: Dr. Marcus Schmidt, Department of Obstetrics and Gynecology, University Medical Center Mainz, \\ Langenbeckstr 1, Mainz 55131, Germany. E-mail: marcus.schmidt@unimedizin-mainz.de \\ How to cite this article: Heimes AS, Schmidt M. Immuno-oncology in triple-negative breast cancer. J Cancer Metastasis Treat \\ 2021;7:9. http://dx.doi.org/10.20517/2394-4722.2020.124
}

Received: 7 Nov 2020 First Decision: 24 Dec 2020 Revised: 25 Dec 2020 Accepted: 6 Jan 2021 Published: 3 Feb 2021

Academic Editor: William Schiemann Copy Editor: Yue-Yue Zhang Production Editor: Xi-Jun Chen

\begin{abstract}
The immune system plays an important role in breast cancer. Triple-negative breast cancer (TNBC) has a higher mutational load compared to other subtypes. In addition, higher levels of tumor-associated antigens suggests that immunotherapies are a promising treatment option especially for TNBC. Our review discusses both the complexity of the immune system and the cancer immune-cell cycle. In fact, a higher level of tumor-infiltrating lymphocytes is associated with an improved prognosis as well as a better response to chemotherapy in TNBC. Important target structures within the cancer immune-cell cycle are the so-called "immune checkpoints". Immune checkpoint inhibitors (ICPi) block the interaction of certain cell surface proteins that serve as "brakes" of immune reactions. Recent studies have shown ICPi improved survival in early as well as advanced TNBC. However, this has the price of increasing, mainly, immune-mediated toxicity. ICPi strengthen tumor-specific T cell-mediated immunity by "releasing the brake" of the immune system. In combination with chemotherapy, ICPi are already approved for TNBC. As a further step, individualized vaccination strategies against tumor-associated neoantigens represent another promising approach. A liposome-formulated intravenous RNA vaccine encoding different tumorassociated antigens is currently being studied in TNBC and leads to neoantigen-specific immune responses. These novel strategies will improve the prognosis of patients with triple-negative breast cancer.
\end{abstract}

Keywords: Tumor infiltrating lymphocytes, cancer-immunity-cycle, immune checkpoint inhibitors, vaccination, tumor-associated antigens, neoantigens 


\section{INTRODUCTION}

The role of the immune system in breast cancer has been discussed for decades ${ }^{[1]}$. Especially triple-negative breast cancer (TNBC), which has a more pronounced immunogenic potential compared to other molecular subtypes, has become a focus of interest. TNBC accounts for $15 \%-20 \%$ of breast cancers and is associated with a significantly poorer prognosis in the first few years after diagnosis when compared with other breast cancer subtypes $^{[2]}$. It is now generally accepted that TNBC is not a homogeneous disease but consists of several subtypes (e.g., basal-like 1 and 2, immunomodulatory, mesenchymal, mesenchymal stem-like, and luminal androgen receptor ${ }^{[3]}$. In TNBC, significantly more somatic mutations and neoantigens are detected than in other molecular subtypes, which speaks for an increased immunogenicity ${ }^{[4]}$. Indeed, it could be shown that transcripts of immune cells as well as tumor-infiltrating lymphocytes (TILs) have their strongest prognostic and predictive influence in TNBC.

\section{Prognostic and predictive relevance of tumor infiltrating lymphocytes}

A reproducible favorable prognostic influence of immune cells was shown slightly more than a decade ago using gene expression analyses ${ }^{[5-8]}$. These initially retrospective results were confirmed in numerous studies using gene expression analyses ${ }^{[9]}$ as well as histological detection of TILs in archival tissue of randomized studies ${ }^{[10]}$. Especially in triple-negative breast cancer, there is a close association between TILs and a more favorable prognosis or better response to neoadjuvant chemotherapy ${ }^{[10-15]}$. This significant association of tumor-infiltrating immune cells and TNBC is not surprising when considering that the total mutational burden is highest in $\mathrm{TNBC}^{[4]}$. In addition, these authors also found that the mutational burden was highly correlated with the neoepitope load $\left(\mathrm{R}^{2}=0.86\right)$. Indeed, an exhaustive analysis of immunogenic signatures in TNBC based on two large-scale breast cancer genomic datasets showed that TNBC had the strongest immunogenicity among breast cancer subtypes ${ }^{[16]}$. Furthermore, these authors also confirmed that TNBC has a higher degree of immune cell infiltration and a higher expression of genes encoding for immune checkpoints than non-TNBC. However, mutational and neoantigen load seem to only incompletely explain immune responses in TNBC since other studies have described an inverse association between immune infiltration and somatic copy number alterations ${ }^{[17,18]}$. Obviously, the exact relationship among immune infiltration, mutational load, and neoantigen load is not yet fully understood. Nonetheless, TILs are widely used especially in TNBC. To improve reproducibility, a standardized methodology for evaluating TILs was defined to integrate this parameter in standard histopathological practice ${ }^{[19]}$. However, TILs are not yet regularly used in routine diagnostics.

\section{COMPLEXITY OF THE IMMUNE SYSTEM}

The role of the immune system in the breast cancer microenvironment is Janus-faced. In addition to tumorinhibiting acute inflammation orchestrated by type 1 T-helper cells (TH1) through CD8 lymphocytes, $\mathrm{B}$ cells, or M1 macrophages, tumor-promoting, TH2-directed chronic inflammation through M2 macrophages, regulatory T-cells, or immune checkpoints such as programmed cell death protein 1 (PD-1) or its ligand programmed cell death 1 ligand 1 (PD-L1) can also occur. Furthermore, bone marrow-derived cells such as myeloid-derived suppressor cells and mesenchymal stromal cells can exert pro-tumorigenic effects through negative regulation of immune responses ${ }^{[20]}$. The immunoediting, i.e., the dynamic interaction between the immune system and the tumor, leads to different stages of tumor evolution (elimination-equilibrium-escape $)^{[21]}$.

\section{Cancer-immunity cycle}

To achieve the destruction of cancer cells by the immune system, different stages must be passed through step by step, which are collectively referred to as the cancer-immunity cycle [Figure 1$]^{[22]}$. 


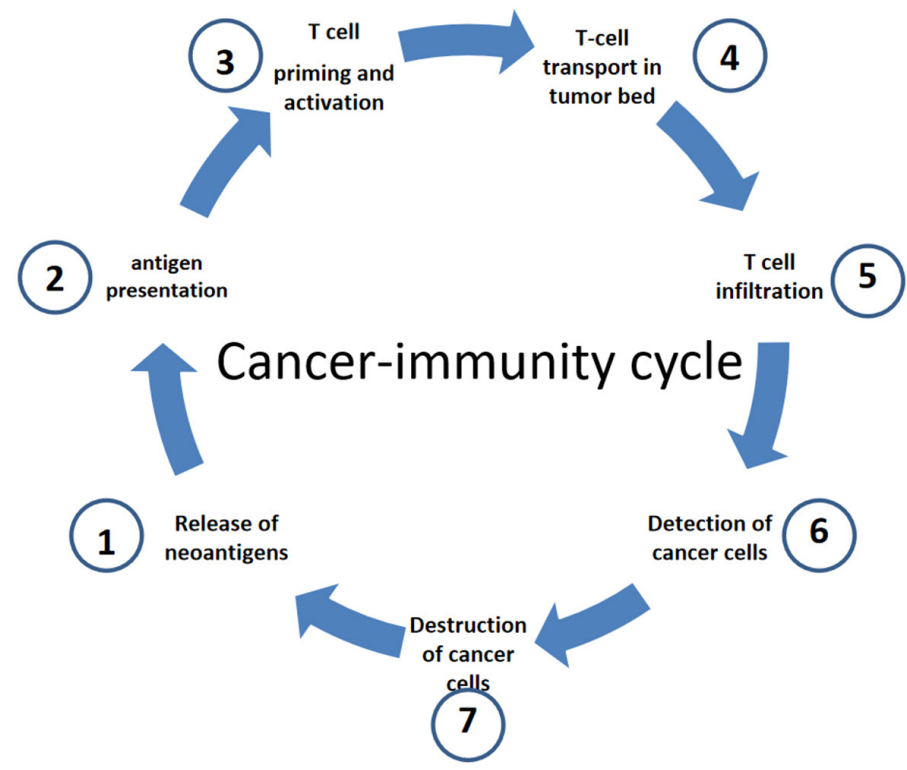

Figure 1. Cancer-immunity cell cycle (modified from ${ }^{[22]}$ ). In a first step, neoantigens are released by cancer cells (1). These neoantigens are then absorbed by dendritic cells (DC). Subsequently, DCs present the antigens to the T cells on major histocompatibility Complex (MHC) I and MHC II molecules (2). This leads to activation of T-cell responses against cancer-specific antigens (3) that are considered foreign or for which central tolerance is incomplete. The activated T cells are then transported into the tumor bed (4) where they infiltrate the tumor (5). Once there, the T cells can specifically recognize (6) and destroy (7) cancer cells through the interaction between their T cell receptor (TCR) and the tumor-specific antigens bound to MHC I. The destruction of the cancer cells releases additional tumor-associated antigens (1), which in turn strengthens the immune response.

Even though the cancer-immunity cycle is only partially functional in many patients with malignant tumors, it is a finely balanced interplay of stimulating and inhibitory signals in order to trigger an effective immune reaction on the one hand and to prevent an excessive immune reaction leading to the development of autoimmunity on the other hand.

\section{Immune checkpoint inhibitors}

Important target structures within the cancer immune-cell cycle are the so-called "immune checkpoints". Immune checkpoint inhibitors (ICPi) block the interaction of certain cell surface proteins that serve as "brakes" of immune reactions. Currently, the most relevant immune checkpoint in breast cancer is PD-1/ $\mathrm{PD}-\mathrm{L} 1{ }^{[23,24]}$. The reaction of PD-1 on T cells with PD-L1, which can be expressed on both $\mathrm{T}$ cells and tumor cells, leads to inhibition of the T cell-mediated immunity against the tumor. However, mechanisms underlying PD-1 upregulation in tumor-infiltrating T cells are not completely understood. For instance, Liu et al. ${ }^{[25]}$ showed that tumor-repopulating cells lead to PD-1 upregulation in CD8-positive T cells through a transcellular kynurenine (Kyn)-aryl hydrocarbon receptor (AhR) pathway. Thus, tumor-repopulating cells escape immune-mediated killing by inducing high PD-1 expression through an IDO-Kyn-AhR-dependent mechanism. Importantly, a pharmacological inhibition of this pathway might also be an efficacious alternative strategy for targeting PD-1 in cancer. In addition, the A2A adenosine receptor is an important negative regulator of immune cells in protecting normal tissues from inflammatory damage ${ }^{[26]}$. Again, there are several pharmacological strategies using selective antagonists to block this pathway and thereby increase anti-tumor immunity ${ }^{[27]}$.

However, at present, monoclonal antibodies, so-called immune checkpoint inhibitors, which block PD-1 or PD-L1 (e.g., pembrolizumab, nivolumab, or atezolizumab) are increasingly used to "release the brake" of the immune system and thus to increase the activity of the immune system against the tumor. The monoactivity of ICPi such as atezolizumab or pembrolizumab was present but manageable in Phase I studies in 
advanced and extensively pretreated $\mathrm{TNBC}^{[21]}$. Especially in less heavily pretreated patients, few but longlasting responses were noticed ${ }^{[28]}$. In a Phase III trial (KEYNOTE-119) in pretreated advanced TNBC, pembrolizumab monotherapy did not significantly improve overall survival compared with single agent chemotherapy, although the pembro treatment effect increased as PD-L1 enrichment increased ${ }^{[29]}$. However, the effectiveness can be significantly increased by adding chemotherapy. Indeed, chemotherapy can lead to immunogenic cell death, which in turn activates the antitumor immune response ${ }^{[30]}$. The combination of immunotherapy with chemotherapy should therefore achieve additive or synergistic clinical activity ${ }^{[31]}$.

Due to the specific mode of action of immune therapies, which in contrast to cytotoxic chemotherapy have no direct effect on tumor cell proliferation, a therapeutic response may be expected at a later stage. In addition, the infiltration of immune cells can lead to an initial increase in the size of the metastases, a so-called pseudoprogression. However, this pseudoprogression occurs in less than $10 \%$ of cases, while, conversely, a very rapid increase in size, a so-called hyperprogression, can occur in up to $29 \%$ of cases. Continuation of therapy with ICPi with imaging evidence of progression should therefore only be considered if the clinical condition has improved and there are no treatment-related toxicities ${ }^{[32]}$.

\section{ICPi in advanced breast cancer}

A Phase I study evaluated the safety and clinical activity of the PD-L1 antibody atezolizumab in 116 patients with metastatic, extensively pretreated $\mathrm{TNBC}^{[33]}$. PD-L1 expression was evaluated immunohistochemically on immune cells as well as on tumor cells. The clinical response rate was determined in the whole cohort, as well as PD-L1 expression and immune cell infiltration within defined subgroups depending on the therapy line. The clinical response rate within the total cohort was $10 \%$. Progression-free survival (PFS) was 1.2 months and overall survival (OS) 17.6 months. Response rates and overall survival were significantly higher in the subgroup with PD-L1-positive immune cells as well as in the first line therapy. The number of TILs was shown to be an independent prognostic and predictive marker and correlated with longer overall survival and better clinical response rates.

For the PD-1 antibody pembrolizumab, several early phase studies showed the efficacy and tolerability as monotherapy. Nanda and co-workers showed in 32 heavily pretreated TNBC patients enrolled in the Phase IB KEYNOTE-012 study that pembrolizumab had an overall response rate of $18.5 \%$ and a median time to response of 17.9 weeks with an acceptable safety profile ${ }^{[34]}$. Building on these encouraging findings, pembrolizumab was evaluated in the Phase II study KEYNOTE-086. In Cohort A, pembrolizumab was evaluated as second or later line of treatment for patients with advanced $\mathrm{TNBC}^{[35]}$. In total, $170 \mathrm{TNBC}$ patients were enrolled; the objective response rate (ORR) was $5.3 \%$ in all patients and $5.7 \%$ in PD-L1positive patients. Median OS was 9.0 months with $12.9 \%$ Grade $3 / 4$ treatment-related adverse events (AE). In Cohort B, 84 PD-L1-positive advanced TNBC patients were treated with $200 \mathrm{mg}$ pembrolizumab as firstline therapy ${ }^{[28]}$. ORR was $21.4 \%$, median duration of response was 10.4 months, and median OS was 18.0 months with 9.3\% Grade 3 adverse events. The authors concluded that pembrolizumab monotherapy had a manageable safety profile and showed durable antitumor activity as first-line therapy for patients with PDL1-positive metastatic TNBC.

In the adaptive, non-comparative Phase II trial TONIC, Voorwerk et al. ${ }^{[36]}$ evaluated several strategies (e.g., irradiation, low-dose cyclophosphamide, cisplatin, and doxorubicin) to make the tumor microenvironment more sensitive to PD-1 blockade with nivolumab in 67 patients with advanced TNBC. In the whole cohort, the objective response rate was $20 \%$. The majority of responses were noticed in the doxorubicin (35\%) and the cisplatin $(23 \%)$ cohorts. Interestingly, the authors noticed in these two cohorts an upregulation of immune-related genes and speculated that this induction approach might induce a more favorable tumor microenvironment and increase the likelihood of response to PD-1 blockade in TNBC. 
Another approach to increase the efficacy of immune checkpoint blockade in advanced cancer is the combination with poly (ADP-ribose) polymerase inhibitors (PARPi). In the MEDIOLA basket trial, durvalumab and olaparib were combined in solid tumors ${ }^{[37]}$. This combination showed promising antitumor activity and safety. Twenty-four of 30 evaluable patients (80\%) had disease control at 12 weeks. While the above combinations are interesting and promising, the combination of ICPi with chemotherapy is currently the most straightforward approach. In a Phase Ib study, the safety and clinical activity of atezolizumab in combination with nanoparticle albumin-bound (nab)-paclitaxel was evaluated in a cohort of 33 extensively pretreated patients with advanced $\mathrm{TNBC}^{[38]}$. The rationale for combining an immune checkpoint inhibitor with chemotherapy is a postulated stronger activation of $\mathrm{T}$ cell-mediated immunity due to an increased release of tumor-associated antigens and resulting immunogenic cell death ${ }^{[31]}$. Primary endpoints of the study were safety and dose finding. Of the 33 patients enrolled in the study, 24 (73\%) developed Grade 3/4 toxicities. The secondary endpoint was the clinical activity of the combination of chemotherapy and ICPi. The ORR within the total cohort was $39.4 \%$ with a median duration of 9.1 months. The PFS was 5.5 months and the OS 14.7 months. The combination with nab-paclitaxel did not lead to an impairment of immune activation by atezolizumab. The results of the study show that the combination of ICPi and nab-paclitaxel is an effective treatment option with a tolerable side effect profile for patients with metastatic TNBC.

Building on these encouraging results, the Phase III study IMpassion130 confirmed the clinical efficacy of atezolizumab in combination with nab-paclitaxel as first-line therapy in a cohort of 902 patients with metastatic or locally advanced $\mathrm{TNBC}^{[39]}$. Patients were randomized to either the experimental arm (atezolizumab combined with nab-paclitaxel) or the placebo arm (nab-paclitaxel + placebo) in a 1:1 ratio. The results show significantly prolonged PFS in both the intention to treat (ITT) population and the PDL1-positive subgroup: PFS was 7.2 months in the experimental arm compared to 5.5 months in the placebo arm [hazard ratio (HR) 0.80; 95\% confidence interval (CI): 0.69-0.92; $P=0.002$ ]. In the subgroup of PDL1-positive ( $\geq 1 \%$ of immune cells) TNBC patients, PFS was 7.5 months compared to five months in the placebo arm. Atezolizumab in combination with nab-paclitaxel prolonged OS in PD-L1-positive patients (25.0 months vs. 15.5 months). Based on these results, atezolizumab in combination with nab-paclitaxel is now approved as first-line therapy in advanced PD-L1-positive TNBC. In a recent analysis of IMpassion130, Schmid et al. ${ }^{[40]}$ showed that atezolizumab did not significantly increase OS in the total cohort from 18.7 to 21 months at longer follow-up (HR 0.86; 95\%CI: 0.72-1.02; $P=0.078$ ). In PD-L1-positive patients, however, OS was prolonged from 18 to 25 months (HR 0.71; 95\%CI: 0.54-0.94). The authors concluded that a clinically meaningful overall survival benefit with atezolizumab plus nab-paclitaxel was found in patients with PD-L1 immune cell-positive disease. Furthermore, they postulated that atezolizumab plus nab-paclitaxel is an important therapeutic option in a disease with high unmet need. Surprisingly, the recently presented IMpassion 131 study, which combined atezolizumab with conventional paclitaxel in advanced TNBC, did not improve PFS or OS vs. placebo + paclitaxe ${ }^{[41]}$. Potential reasons for this obvious contrast with the benefit seen in IMpassion130 still need further exploration, although several hypotheses (e.g., differences within the study population as well as different taxanes and the role of steroids) are under discussion ${ }^{[42]}$.

At the annual meeting of the American Society of Clinical Oncology, Cortes et al. ${ }^{[43]}$ presented results of KEYNOTE-355, a randomized, double-blind, Phase III study of pembrolizumab + chemotherapy versus placebo + chemotherapy for previously untreated locally recurrent inoperable or metastatic triplenegative breast cancer. They showed that pembrolizumab combined with several chemotherapy partners (nab-paclitaxel, paclitaxel, or gemcitabine/carboplatin) showed a statistically significant and clinically meaningful improvement in PFS versus chemotherapy alone in patients with previously untreated locally recurrent inoperable or metastatic TNBC whose tumors expressed PD-L1. Furthermore, pembrolizumab + chemotherapy was generally well tolerated, with no new safety concerns. This study was recently published in full ${ }^{[4]}$. Based on their findings, the authors concluded that the addition of pembrolizumab to standard 
Table 1. Randomized evidence Phase III for ICPi in advanced triple-negative breast cancer

\begin{tabular}{|c|c|c|c|c|}
\hline Ref. & $n$ & Therapy & PFS in ITT & OS in ITT \\
\hline Schmid et al. ${ }^{[39]}$, NEJM 2018 & 902 & Nab-Paclitaxel \pm Atezolizumab & $\begin{array}{l}7.2 \mathrm{~m} \text { vs. } 5.5 \mathrm{~m} \\
\text { HR } 0.80(0.69-0.92)\end{array}$ & $\begin{array}{l}21.3 \mathrm{~m} \text { vs. } 17.6 \mathrm{~m} \\
\text { HR } 0.84(0.69-1.02)\end{array}$ \\
\hline Miles et al. ${ }^{[41]}$, ESMO 2020 & 943 & Paclitaxel \pm Atezolizumab & $\begin{array}{l}5.7 \mathrm{~m} \text { vs. } 5.6 \mathrm{~m} \\
\text { HR } 0.86(0.70-1.05)\end{array}$ & $\begin{array}{l}19.2 \text { m vs. } 22.8 \text { m } \\
\text { HR } 1.11 \text { (0.87-1.42) }\end{array}$ \\
\hline Cortes et al. ${ }^{[44]}$, Lancet 2020 & 847 & Chemotherapy \pm pembrolizumab & $\begin{array}{l}7.5 \mathrm{~m} \text { vs. } 5.6 \mathrm{~m} \\
\text { HR } 0.82(0.69-0.97)\end{array}$ & \\
\hline Cortés et al. ${ }^{[29]}$, ESMO 2019 & 622 & Pembrolizumab vs. chemotherapy & $\begin{array}{l}2.1 \mathrm{~m} \text { vs. } 3.3 \mathrm{~m} \\
\text { HR } 1.60(1.33-1.92)\end{array}$ & $\begin{array}{l}9.9 \mathrm{~m} \text { vs. } 10.8 \mathrm{~m} \\
\text { HR } 0.97(0.82-1.15)\end{array}$ \\
\hline
\end{tabular}

HR: Hazard ratio; ICPi: immune-checkpoint inhibitors; ITT: intention to treat; m: months; OS: overall survival; PFS: progression-free survival.

chemotherapy has a role in the first-line treatment of advanced triple-negative breast cancer. The findings of Phase III studies in advanced TNBC are summarized in Table 1.

\section{ICPi in early breast cancer}

Due to the effectiveness of ICPi in advanced breast cancer, studies have also been initiated in early TNBC. In a randomized Phase II study in early TNBC, the PD-L1 antibody durvalumab was combined with an anthracycline- and taxane- containing neoadjuvant chemotherapy (NACT) in 174 TNBC patients ${ }^{[45]}$. Overall, $87 \%$ of the patients were PD-L1-positive. Pathologic complete remission (pCR) was increased from $44.2 \%$ to $53.4 \%$ with durvalumab. A significant increase in pCR was found in the subgroup $(n=117)$ who received neoadjuvant durvalumab for two weeks before the start of NACT (61\% vs. $41.4 \%$; $P=0.035$ ). Immune-mediated thyroid dysfunction occurred in $47 \%$ with overall good tolerability. Interestingly, a pre-planned exploratory analysis of this study showed that both tumor mutational burden (TMB) and an immune gene expression profile (GEP) independently predicted PCR in TNBC patients ${ }^{[46]}$. In patients with both high TMB and GEP, pCR rate was $82 \%$ in contrast to $28 \%$ in the group with both low TMB and GEP. These findings encourage further analysis of TMB in combination with immune parameters to individually tailor therapies in breast cancer.

Furthermore, pembrolizumab in addition to standard taxane- and anthracycline- based NACT was evaluated in the adaptively randomized I-SPY Phase II study ${ }^{[47]}$. In TNBC, pembrolizumab increased pCR from $22 \%$ to $60 \%$ with an acceptable safety profile.

In the neoadjuvant Phase III study KEYNOTE-522, 1174 patients with early TNBC neoadjuvant were treated with anthracycline-, taxane- and platinum- containing chemotherapy \pm pembrolizumab ${ }^{[48]}$. The addition of pembrolizumab significantly increased pCR from $51.2 \%$ to $64.8 \%(P=0.00055)$. This increase in $\mathrm{pCR}$ was observed independent of the PD-L1 status. Additionally, pembrolizumab improved event-free survival (HR 0.63; 95\%CI: 0.43-0.93). Grade 3/4 toxicities were also increased with pembrolizumab (78\% vs. $73 \%)$.

The NeoTRIPaPDL1 Michelangelo randomized study investigated neoadjuvant nab-paclitaxel treatment with or without atezolizumab in triple negative, early high-risk, and locally advanced breast cancer, failing to show a significant increase of pCR with atezolizumab ${ }^{[49]}$. Recently, however, results for efficacy and safety of atezolizumab $v s$. placebo combined with nab-paclitaxel followed by doxorubicin plus cyclophosphamide as neoadjuvant treatment for early-stage TNBC were presented ${ }^{[50]}$. In total, 333 patients with early TNBC were enrolled in the double-blind, randomized, Phase III study IMpassion031. Atezolizumab increased pCR from $41 \%$ to $58 \%$. In the PD-L1-positive population, pCR was increased from $49 \%$ to $69 \%$. Treatmentrelated serious adverse events occurred in $23 \%$ and $16 \%$, respectively. The authors concluded that neoadjuvant treatment with atezolizumab in combination with nab-paclitaxel and anthracycline-based 
Table 2. Randomized Phase III evidence for ICPi in early triple-negative breast cancer

\begin{tabular}{lclll}
\hline Ref. & $\boldsymbol{n}$ & \multicolumn{1}{c}{ Therapy } & \multicolumn{1}{c}{ pCR in ITT } & EFS in ITT \\
\hline Schmid et al. ${ }^{[48]}$, NEJM 2020 & 1174 & Chemotherapy \pm pembrolizumab & $64.8 \%$ vs. 51.2\% & HR 0.63 (0.43-0.93) \\
Mittendorf et al. ${ }^{[50]}$, Lancet 2020 & 333 & Chemotherapy \pm atezolizumab & $58 \%$ vs. 41\% & HR 0.76 (0.40-1.44) \\
Gianni et al. ${ }^{[49]}$, SABCS 2019 & 280 & Chemotherapy \pm atezolizumab & $43.5 \%$ vs. 40.8\% & \\
\hline
\end{tabular}

EFS: Event-free survival; HR: hazard ratio; ICPi: immune-checkpoint inhibitors; ITT: intention to treat; m: months; pCR: pathological complete response.

Table 3. Immune-mediated adverse effects of immune checkpoint inhibitors

\begin{tabular}{|c|c|}
\hline Organ & Symptoms \\
\hline Brain & $\begin{array}{l}\text { Headache, changes in mental state, confusion, depressive mood, sensitivity to light, seizure, } \\
\text { motor or sensory dysfunction, meningitis, stiffness of the neck }\end{array}$ \\
\hline Nerves & $\begin{array}{l}\text { Muscle weakness (including eye muscles), fatigue, difficulty swallowing, paranesthesia or } \\
\text { altered sensory perception, ascending or progressive paralysis, weakness of the respiratory } \\
\text { muscles }\end{array}$ \\
\hline Skin & Persistent and/or severe rash or pruritus \\
\hline $\begin{array}{l}\text { Thyroid gland, adrenal glands, pituitary } \\
\text { gland, pancreatic islets of Langerhans }\end{array}$ & $\begin{array}{l}\text { Fatigue, headache, changes in mental state, heat or cold intolerance, tachycardia or } \\
\text { bradycardia, bowel movement irregularities, weight change, polyuria or polydipsia, blurred } \\
\text { vision }\end{array}$ \\
\hline Lungs & $\begin{array}{l}\text { Difficulty breathing or coughing, radiological changes (e.g., focal ground-glass opacity, spotty } \\
\text { uneven infiltrates), dyspnea, hypoxia }\end{array}$ \\
\hline Liver & $\begin{array}{l}\text { Increase in transaminase values, increase in total bilirubin, jaundice, right-sided abdominal } \\
\text { pain, fatigue }\end{array}$ \\
\hline Pancreas & Stomachache, nausea, vomiting, fever \\
\hline Gut & Aqueous, soft or liquid stool, diarrhea, abdominal pain, mucus or blood in the stool \\
\hline
\end{tabular}

chemotherapy improved pCR in early TNBC patients with an acceptable safety profile. The findings of Phase III studies in early TNBC are summarized in Table 2.

In fact, all these randomized studies using ICPi in early or advanced breast cancer showed a significant advantage over standard therapy alone. In combination with an acceptable safety profile, immune checkpoint inhibitors are a promising new therapeutic option in TNBC.

\section{Predictive markers for immune checkpoint inhibitors}

At present, the only established predictive biomarker for the response to ICPi in advanced TNBC is PDL1 status. Recent analyses show a potential role for tumor mutational load (TMB) for the response to durvalumab in early $\mathrm{TNBC}^{[46]}$. In a recently published comprehensive genomic analysis of 3831 consecutive breast cancer samples, potential biomarkers [e.g., TMB, microsatellite instability (MSI), and BRCA mutations] to guide the use of ICPIs for these patients were evaluated ${ }^{[51]}$. However, for all these potential biomarkers, prospective randomized trials will be necessary to assess the predictive value for the response to immune checkpoint inhibitors.

\section{Adverse events of immune checkpoint inhibitors}

Adverse events of ICPi are mainly explained by the mode of action. ICPi block so-called immune checkpoints, which act as a "brake" on triggered immune reactions.

When this "brake" is blocked by antibodies such as ICPi, an unhindered immune reaction can occur, which can also affect the body's own tissues through autoimmune phenomena. This is associated with a spectrum of side effects related to the mechanism of action. Side effects may affect several organs of the body and are most commonly found in the skin, gastrointestinal tract, lungs, thyroid, adrenal gland, pituitary gland, kidney, nervous system, and musculoskeletal, ocular, or cardiovascular system ${ }^{[52]}$ [Table 3]. During 
Table 4. Management of adverse effects of immune checkpoint inhibitors (modified from ${ }^{[52]}$ )

\begin{tabular}{|c|c|}
\hline CTC & Actions \\
\hline Grade 1 & Continue with close monitoring \\
\hline Grade 2 & Interrupt therapy until improvement to Grade 1, consider corticosteroids if necessary \\
\hline Grade 3 & $\begin{array}{l}\text { Administration of corticosteroids (prednisone } 1-2 \mathrm{mg} / \mathrm{kg} / \text { day or methylprednisolone } 1-2 \mathrm{mg} / \mathrm{kg} / \mathrm{day} \text { ) } \\
\text { Taper off } 4-6 \text { weeks } \\
\text { Infliximab, if no improvement on corticosteroids within } 48-72 \mathrm{~h}\end{array}$ \\
\hline Grade 4 & Termination of therapy (exception: endocrinopathies with improvement through hormone substitution) \\
\hline
\end{tabular}

CTC: Common Toxicity Criteria.

treatment, it is important to note that these immune-mediated side effects may occur after very different time intervals and sometimes even after the end of therapy with ICPi.

\section{Management of adverse events}

Early diagnosis and therapy can reduce the severity and duration of immune-mediated side effects. The proper management of these adverse events is therefore essential. Depending on the severity of the side effects, different measures are recommended. In the case of pronounced worsening of symptoms, therapy with corticosteroids or even termination of treatment with ICPi is required [Table 4$]^{[52]}$.

\section{Vaccination}

Improving the antigen presentation by vaccination to trigger a protective immune response against breast cancer is an obvious strategy. However, vaccinations against solid tumors such as breast cancer have thus far only shown limited efficacy. In most cases, known antigens such as HER2 were used, but these vaccination strategies have not yet shown clinically relevant success ${ }^{[53]}$. A fundamental disadvantage of this approach is that the immune reactions against known self-antigens such as HER2 are mostly weak, since the $\mathrm{T}$ lymphocytes, which have a high affinity against these self-antigens, are subject to central tolerance. Using high-throughput mutation analysis methods such as Next Generation Sequencing (NGS), individual non-synonymous somatic mutations (the so-called mutanome) are increasingly coming into focus ${ }^{[54]}$. These mutations are not subject to central immunological tolerance ${ }^{[55]}$. The resulting neoantigens are therefore ideally suited for individual vaccination. With the help of complex prediction algorithms, which consider among others the MHC binding affinity, the neoantigens with the highest expected immunogenicity are selected from the mutanome of a tumor. The RNA of these neoantigens is used as vaccine and applied intravenously. In a first step, the RNA is translated into the corresponding protein in dendritic cells. The further processing of these neoepitopes is performed on the one hand via the proteasome with subsequent antigen presentation via MHC I and consecutive stimulation of cytotoxic CD8 T cells, which leads to tumor cell destruction. On the other hand, the endosomal route leads to presentation via MHC II with activation of $\mathrm{CD} 4 \mathrm{~T}$ cells ${ }^{[55]}$. While the focus of anti-tumor immunity research has long been on MHC I and CD8 T cells, it could be shown in mouse models that the majority of immunogenic mutations are presented via MHC II and recognized by CD4 $\mathrm{T}$ cells ${ }^{[56]}$. In the meantime, the clinical efficacy of an individual RNA vaccination against the individual mutanome of a tumor in patients with advanced malignant melanoma has been described ${ }^{[57]}$.

Building on these encouraging findings, we conducted a Phase I study in early TNBC after completion of standard (neo)adjuvant chemotherapy [Mutanome Engineered RNA Immuno-Therapy (MERIT) ${ }^{[58]}$. The aim of this study was to demonstrate feasibility, safety, and biological efficacy of a liposome-formulated intravenous RNA vaccine encoding different tumor antigens. Patients received eight intravenous vaccinations, either with a personalized RNA vaccine based on the antigen expression profile of the respective tumor or with an individualized RNA vaccine against up to 20 neoepitopes identified by NGS. Recently, we reported at the annual meeting of the European Society of Medical Oncology preliminary 
results of immune responses in vaccinated patients as analyzed by interferon-gamma (IFN $\gamma$ )-ELISpot, multimerstaining, TCR repertoire profiling, and single cell TCR sequencing ${ }^{[59]}$. A substantial number of T cell responses against individual neoepitopes were induced de novo and of high magnitude. In addition, the T cell response was sustained at high levels for at least six months after the last vaccination. This suggests that the individualized vaccination is highly efficient in inducing strong poly-epitopic T-cell responses in patients with TNBC in the post-(neo)adjuvant setting. In fact, as an important effector cytokine for anticancer immunity, IFN $\gamma$ also has independent prognostic significance in basal-like or tiple-negative breast cancer, which supports a beneficial effect of IFN $\gamma$-mediated immune responses through vaccination ${ }^{[60]}$.

With this vaccination strategy, $\mathrm{T}$ cell responses against tumor-specific neoantigens can be triggered. Such vaccines may lead to an increase in immunogenicity of tumors lacking spontaneous immunogenicity, which should make these tumors more responsive to treatment with ICPi. Therefore, a combination of RNA vaccination with ICPi might be useful to stimulate the body's immune system against tumor cells, including in ICPi-experienced patients ${ }^{[61]}$.

The long-term goal of this individualized vaccination against non-synonymous mutations in early triplenegative breast cancer is to hit the Achilles heel of these tumors and thus significantly improve the prognosis of affected patients.

\section{CONCLUSION}

The immune system plays an important role in triple-negative breast cancer. A high expression of tumorinfiltrating lymphocytes or immune transcripts is associated with an improved prognosis as well as an increased response to chemotherapy. Novel therapies such as immune checkpoint inhibitors have improved survival in triple-negative breast cancer. Management of side effects is of essential importance. Individualized vaccination strategies against the mutanome of a tumor are promising.

\section{DECLARATIONS}

\section{Authors contributions}

Made substantial contributions to the conception and drafted the work: Heimes AS, Schmidt M

\section{Availability of data and materials}

Not applicable.

\section{Financial support and sponsorship}

None.

\section{Conflict of interest}

Schmidt M has been a consultant to Astra Zeneca, Celgene, Eisai, Novartis, Pfizer and Roche and has received consulting fees from these companies as well as from Lilly, Merck, Myelo Therapeutics, Pantarhei Bioscience and Pierre-Fabre. Research support was received from AstraZeneca, BioNTech, Eisai, Genentech, Myelo Therapeutics, Novartis, Pantarhei Bioscience, Pfizer, Pierre-Fabre and Roche. Heimes AS reports no conflict of interest.

\section{Ethical approval and consent to participate}

Not applicable.

\section{Consent for publication}

Not applicable. 


\section{Copyright}

(c) The Author(s) 2021.

\section{REFERENCES}

1. BERG JW. Inflammation and prognosis in breast cancer; a search for host resistance. Cancer 1959;12:714720.

2. Metzger-Filho O, Tutt A, Azambuja E de et al. Dissecting the heterogeneity of triple-negative breast cancer. J Clin Oncol 2012;30:187987.

3. Lehmann BD, Bauer JA, Chen X, et al. Identification of human triple-negative breast cancer subtypes and preclinical models for selection of targeted therapies. J Clin Invest 2011;121:2750-67.

4. Narang P, Chen MX, Sharma AA, Anderson KS, Wilson MA. The neoepitope landscape of breast cancer: implications for immunotherapy. BMC Cancer 2019;19:200.

5. Teschendorff AE, Miremadi A, Pinder SE, Ellis IO, Caldaset C. An immune response gene expression module identifies a good prognosis subtype in estrogen receptor negative breast cancer. Genome Biol 2007;8:R157.

6. Alexe G, Dalgin GS, Scanfeld D, et al. High expression of lymphocyte-associated genes in node-negative HER2+ breast cancers correlates with lower recurrence rates. Cancer Res 2007;67:10669-76.

7. Schmidt M, Bohm D, von Torne C, et al. The humoral immune system has a key prognostic impact in node-negative breast cancer. Cancer Res 2008;68:5405-13.

8. Rody A, Holtrich U, Pusztai L, et al. T-cell metagene predicts a favorable prognosis in estrogen receptor-negative and HER2-positive breast cancers. Breast Cancer Res 2009;11:R15.

9. Schmidt M, Weyer-Elberich V, Hengstler JG, et al. Prognostic impact of CD4-positive T cell subsets in early breast cancer: a study based on the FinHer trial patient population. Breast Cancer Res 2018;20:15.

10. Denkert C, Minckwitz G von, Darb-Esfahani S, et al. Tumour-infiltrating lymphocytes and prognosis in different subtypes of breast cancer: a pooled analysis of 3771 patients treated with neoadjuvant therapy. Lancet Oncol 2018;19:40-50.

11. Adams S, Gray RJ, Demaria S, et al. Prognostic value of tumor-infiltrating lymphocytes in triple-negative breast cancers from two phase III randomized adjuvant breast cancer trials: ECOG 2197 and ECOG 1199. J Clin Oncol 2014;32:2959-66.

12. Loi S, Sirtaine N, Piette F, et al. Prognostic and predictive value of tumor-infiltrating lymphocytes in a phase III randomized adjuvant breast cancer trial in node-positive breast cancer comparing the addition of docetaxel to doxorubicin with doxorubicin-based chemotherapy: BIG 02-98. J Clin Oncol 2013;31:860-7.

13. Loi S, Michiels S, Salgado R, et al. Tumor infiltrating lymphocytes are prognostic in triple negative breast cancer and predictive for trastuzumab benefit in early breast cancer: results from the FinHER trial. Ann Oncol 2014;25:1544-50.

14. Ibrahim EM, Al-Foheidi ME, Al-Mansour MM, Kazkaz GA. The prognostic value of tumor-infiltrating lymphocytes in triple-negative breast cancer: a meta-analysis. Breast Cancer Res Treat 2014;148:467-76.

15. Hida AI, Watanabe T, Sagara Y, et al. Diffuse distribution of tumor-infiltrating lymphocytes is a marker for better prognosis and chemotherapeutic effect in triple-negative breast cancer. Breast Cancer Res Treat 2019;178:283-94.

16. Liu ZX, Li MY, Jiang ZH, Wang XS. A comprehensive immunologic portrait of triple-negative breast cancer. Transl Oncol 2018;11:31129.

17. Karn T, Jiang T, Hatzis C, et al. Association between genomic metrics and immune infiltration in triple-negative breast cancer. JAMA Oncol 2017;3:1707-11.

18. Safonov A, Jiang T, Bianchini G, et al. Immune gene expression is associated with genomic aberrations in breast cancer. Cancer Res 2017;77:3317-24.

19. Salgado R, Denkert C, Demaria S, et al. The evaluation of tumor-infiltrating lymphocytes (TILs) in breast cancer: recommendations by an International TILs Working Group 2014. Ann Oncol 2015;26:259-71.

20. Bianchi G, Borgonovo G, Pistoia V, Raffaghello L. Immunosuppressive cells and tumour microenvironment: focus on mesenchymal stem cells and myeloid derived suppressor cells. Histol Histopathol 2011;26:941-51.

21. Emens LA. Breast cancer immunotherapy: facts and hopes. Clin Cancer Res 2018;24:511-20.

22. Chen DS, Mellman I. Oncology meets immunology: the cancer-immunity cycle. Immunity 2013;39:1-10.

23. Postow MA, Callahan MK, Wolchok JD. Immune checkpoint blockade in cancer therapy. J Clin Oncol 2015;33:1974-82.

24. Ribas A. Releasing the brakes on cancer immunotherapy. N Engl J Med 2015;373:1490-2.

25. Liu YY, Liang XY, Dong WQ, et al. Tumor-repopulating cells induce PD-1 expression in CD8(+) T cells by transferring kynurenine and AhR activation. Cancer Cell 2018;33:480-94.e7.

26. Ohta A, Gorelik E, Prasad SJ, et al. A2A adenosine receptor protects tumors from antitumor T cells. Proc Natl Acad Sci U S A 2006;103:13132-7.

27. Zhang JF, Yan WZ, Duan WW, Wüthrich K, Cheng JJ. Tumor immunotherapy using A(2A) adenosine receptor antagonists. Pharmaceuticals (Basel) 2020;13:237.

28. Adams S, Loi S, Toppmeyer D, et al. Pembrolizumab monotherapy for previously untreated, PD-L1-positive, metastatic triple-negative breast cancer: cohort B of the phase II KEYNOTE-086 study. Ann Oncol 2019;30:405-11.

29. Cortés J, Lipatov O, Im SA, et al. KEYNOTE-119: phase III study of pembrolizumab (pembro) versus single-agent chemotherapy (chemo) for metastatic triple negative breast cancer (mTNBC). Annals of Oncology 2019;30:v859-60.

30. Andre F, Dieci MV, Dubsky P, et al. Molecular pathways: involvement of immune pathways in the therapeutic response and outcome in 
breast cancer. Clin Cancer Res 2013;19:28-33.

31. Emens LA, Middleton G. The interplay of immunotherapy and chemotherapy: harnessing potential synergies. Cancer Immunol Res 2015;3:436-43.

32. Borcoman E, Kanjanapan Y, Champiat S, et al. Novel patterns of response under immunotherapy. Ann Oncol 2019;30:385-96.

33. Emens LA, Cruz C, Eder JP, et al. Long-term clinical outcomes and biomarker analyses of atezolizumab therapy for patients with metastatic triple-negative breast cancer: a phase 1 study. JAMA Oncol 2019;5:74-82.

34. Nanda R, Chow LQM, Dees EC, et al. Pembrolizumab in Patients With Advanced Triple-Negative Breast Cancer: Phase Ib KEYNOTE-012 Study. J Clin Oncol 2016;34:2460-7.

35. Adams S, Schmid P, Rugo HS, et al. Pembrolizumab monotherapy for previously treated metastatic triple-negative breast cancer: cohort A of the phase II KEYNOTE-086 study. Ann Oncol 2019;30:397-404.

36. Voorwerk L, Slagter M, Horlings HM, et al. Immune induction strategies in metastatic triple-negative breast cancer to enhance the sensitivity to PD-1 blockade: the TONIC trial. Nat Med 2019;25:920-8.

37. Domchek SM, Postel-Vinay S, Im SA, et al. Olaparib and durvalumab in patients with germline BRCA-mutated metastatic breast cancer (MEDIOLA): an open-label, multicentre, phase 1/2, basket study. Lancet Oncol 2020;21:1155-64.

38. Adams S, Diamond JR, Hamilton E, et al. Atezolizumab plus nab-Paclitaxel in the treatment of metastatic triple-negative breast cancer with 2-year survival follow-up: a phase 1b clinical trial. JAMA Oncol 2019;5:334-42.

39. Schmid P, Adams S, Rugo HS, et al. Atezolizumab and nab-paclitaxel in advanced triple-negative breast cancer. $N$ Engl $J$ Med 2018;379:2108-21.

40. Schmid P, Rugo HS, Adams S, et al. Atezolizumab plus nab-paclitaxel as first-line treatment for unresectable, locally advanced or metastatic triple-negative breast cancer (IMpassion130): updated efficacy results from a randomised, double-blind, placebo-controlled, phase 3 trial. Lancet Oncol 2020;21:44-59.

41. Miles D, Andre F, Gligorov J, et al. IMpassion131: phase III study comparing 1L atezolizumab with paclitaxel vs placebo with paclitaxel in treatment-naive patients with inoperable locally advanced or metastatic triple negative breast cancer (mTNBC). Ann Oncol 2017;28:v105.

42. Franzoi MA, Azambuja E de. Atezolizumab in metastatic triple-negative breast cancer: IMpassion130 and 131 trials - how to explain different results? ESMO Open 2020;5:e01112.

43. Cortes J, Cescon DW, Rugo HS, et al. KEYNOTE-355: randomized, double-blind, phase III study of pembrolizumab + chemotherapy versus placebo + chemotherapy for previously untreated locally recurrent inoperable or metastatic triple-negative breast cancer. $J$ Clin Oncol 2020;38:1000.

44. Cortes J, Cescon DW, Rugo HS, et al. Pembrolizumab plus chemotherapy versus placebo plus chemotherapy for previously untreated locally recurrent inoperable or metastatic triple-negative breast cancer (KEYNOTE-355): a randomised, placebo-controlled, double-blind, phase 3 clinical trial. Lancet 2020;396:1817-28.

45. Loibl S, Untch M, Burchardi N, et al. A randomised phase II study investigating durvalumab in addition to an anthracycline taxanebased neoadjuvant therapy in early triple-negative breast cancer: clinical results and biomarker analysis of GeparNuevo study. Ann Oncol 2019;30:1279-88.

46. Karn T, Denkert C, Weber KE, et al. Tumor mutational burden and immune infiltration as independent predictors of response to neoadjuvant immune checkpoint inhibition in early TNBC in GeparNuevo. Ann Oncol 2020;31:1216-22.

47. Nanda R, Liu MC, Yau C et al. Effect of Pembrolizumab Plus Neoadjuvant Chemotherapy on Pathologic Complete Response in Women With Early-Stage Breast Cancer: An Analysis of the Ongoing Phase 2 Adaptively Randomized I-SPY2 Trial. JAMA Oncol 2020;6:676-84.

48. Schmid P, Cortes J, Pusztai L, et al. Pembrolizumab for early triple-negative breast cancer. $N$ Engl J Med 2020;382:810-21.

49. Gianni L, Huang CF, Egle D, et al. Abstract GS3-04: pathologic complete response (pCR) to neoadjuvant treatment with or without atezolizumab in triple negative, early high-risk and locally advanced breast cancer. NeoTRIPaPDL1 Michelangelo randomized study 2020;GS3-04.

50. Mittendorf EA, Zhang H, Barrios CH, et al. Neoadjuvant atezolizumab in combination with sequential nab-paclitaxel and anthracyclinebased chemotherapy versus placebo and chemotherapy in patients with early-stage triple-negative breast cancer (IMpassion031): a randomised, double-blind, phase 3 trial. Lancet 2020;396:1090-100.

51. Sivapiragasam A, Ashok Kumar P, Sokol ES, et al. Predictive biomarkers for immune checkpoint inhibitors in metastatic breast cancer. Cancer Med 2020.

52. Brahmer JR, Lacchetti C, Schneider BJ, et al. Management of immune-related adverse events in patients treated with immune checkpoint inhibitor therapy: american society of clinical oncology clinical practice guideline. J Clin Oncol 2018;36:1714-68.

53. Peoples GE, Gurney JM, Hueman MT, et al. Clinical trial results of a HER2/neu (E75) vaccine to prevent recurrence in high-risk breast cancer patients. J Clin Oncol 2005;23:7536-45.

54. Castle JC, Kreiter S, Diekmann J, et al. Exploiting the mutanome for tumor vaccination. Cancer Res 2012;72:1081-91.

55. Tureci O, Vormehr M, Diken M, et al. Targeting the heterogeneity of cancer with individualized neoepitope vaccines. Clin Cancer Res 2016;22:1885-96.

56. Kreiter S, Vormehr M, van de Roemer N, et al. Mutant MHC class II epitopes drive therapeutic immune responses to cancer. Nature 2015;520:692-6.

57. Sahin U, Derhovanessian E, Miller M, et al. Personalized RNA mutanome vaccines mobilize poly-specific therapeutic immunity against cancer. Nature 2017;547:222-6.

58. Schmidt M, Bolte S, Frenzel K, et al. Abstract OT2-06-01: highly innovative personalized RNA-immunotherapy for patients with triple 
negative breast cancer. Cancer Res 2019;79:OT2-06.

59. Schmidt M, Vogler I, Derhovanessian E, et al. 88MO T-cell responses induced by an individualized neoantigen specific immune therapy in post (neo)adjuvant patients with triple negative breast cancer. Ann Oncol 2020;31:S276.

60. Heimes A-S, Härtner F, Almstedt K, et al. Prognostic significance of interferon- $\gamma$ and its signaling pathway in early breast cancer depends on the molecular subtypes. Int J Mol Sci 2020;21:7178.

61. Sahin U, Oehm P, Derhovanessian E, et al. An RNA vaccine drives immunity in checkpoint-inhibitor-treated melanoma. Nature 2020;585:107-12. 